\title{
Multiobjective Design Optimization Using Dual-Level Response Surface Methodology and Booth's Algorithm for Permanent Magnet Synchronous Generators
}

\author{
Pedram Asef ${ }^{(}$, Student Member, IEEE, Ramon Bargalló Perpiñà ${ }^{(}$, M. R. Barzegaran $\left.{ }^{(}\right)$, Member, IEEE, \\ Andrew Lapthorn ${ }^{\mathbb{E}}$, Member, IEEE, and Daniela Mewes, Student Member, IEEE
}

\begin{abstract}
This paper studies a dual-level response surface methodology (DRSM) coupled with Booth's algorithm using a simulated annealing (BA-SA) method as a multiobjective technique for parametric modeling and machine design optimization for the first time. The aim of the research is for power maximization and cost of manufacture minimization resulting in a highly optimized wind generator to improve small power generation performance. The DRSM is employed to determine the best set of design parameters for power maximization in a surface-mounted permanent magnet synchronous generator with an exterior-rotor topology. Additionally, the BA-SA method is investigated to minimize material cost while keeping the volume constant. DRSM by different design functions including mixed resolution robust design, full factorial design, central composite design, and box-behnken design are applied to optimize the power performance resulting in very small errors. An analysis of the variance via multilevel RSM plots is used to check the adequacy of fit in the design region and determines the parameter settings to manufacture a high-quality wind generator. The analytical and numerical calculations have been experimentally verified and have successfully validated the theoretical and multiobjective optimization design methods presented.
\end{abstract}

Index Terms-Dual response surface methodology, Booth's algorithm, synchronous machine, finite element analysis, multiobjective optimization.

\section{INTRODUCTION}

$\mathbf{I}$ $\mathrm{N}$ THE process of optimizing electrical machines, the major objective is to define a set of key design parameters that include geometry, topology, and material choice. These appease

Manuscript received March 27, 2017; revised July 19, 2017 and September 18, 2017; accepted November 19, 2017. Date of publication November 22, 2017; date of current version May 17, 2018. Paper no. TEC-00229-2017. (Corresponding author: M. R. Barzegaran.)

P. Asef and R. B. Perpiñà are with the Department of Electrical Engineering, Polytechnic University of Catalonia-BarcelonaTech, EEBE, Barcelona 08019, Spain (e-mail: pedram.asef@upc.edu; ramon.bargallo@upc.edu).

M. R. Barzegaran is with the Renewable Energy Microgrid Laboratory, Lamar University (A Texas State University), Beaumont, TX 77705 USA (e-mail: barzegaran@lamar.edu)

A. Lapthorn is with the Department of Electrical and Computer Engineering, University of Canterbury, Christchurch 8041, New Zealand (e-mail: andrew. lapthorn@canterbury.ac.nz).

D. Mewes is with the KTH Royal Institute of Technology, Industrial, Engineering and Management, Stockholm 100-44, Sweden (e-mail: mewes@kth.se).

Color versions of one or more of the figures in this paper are available online at http://ieeexplore.ieee.org.

Digital Object Identifier 10.1109/TEC.2017.2777397 specified design specifications and constraints, such as output power, which is observed as a major concern. In this paper, the research objectives focus on developing an application-oriented, design optimization method based on deterministic and optimal design methods due to their reliability, simplicity, and practicality. The robust design is investigated in different subject domains such as numerical analysis (FEA) and analytical models for the evaluation of performance.

Recently, SPMSGs have been employed for power generation to implement high-power, high-torque, high-efficiency, smallscale, and low-maintenance systems. The PM generator applied to conventional small-scale wind power generation has been designed with an exterior rotor topology because its structure has the desired advantage to generate power with higher output power density at low speeds which are often found in urban applications [1].

The DRSM, as a multi-level empirical modeling method, is investigated for the modeling and analysis of problems in which a number of variables (treatments) impact the observed result; and where the objective is to optimize the quantity of interest. This also avoids the time-consuming drawbacks of direct simulation which result in significant costs. The methodology is particularly applicable to the design of yields or processes, which can be sensitive to uncontrollable variables. A local and global sensitivity analysis is necessary to consider for the noise variables in the design. Then, a combination of settings can be applied to the control variables, which allows the process output to remain robust and flexible to changes in the noise variables.

In terms of the optimization process and yielded design, there are many approaches, which fit the robust design requirements. The mixed resolution robust design (known as MR-RD) has been observed to decrease the number of experimental runs, and is compared to the FFD approach. A conceptual study of this surface methodology was reviewed by D. C. Montgomery [2]. G. Lei et al. [3] have studied how to apply multidisciplinary machine design optimization using RSM. A very good mathematical basic modelling of RSM in electrical machines has been presented in these references.

L. Jolly et al. [4] presented how the process of integrating a single level RSM with a genetic algorithm can be applied to a multi-objective optimization. This study dealt with a newer and common CCD function to estimate the optimum operation point at a PMSM. This work suffers a comparison among all design functions used, and also experimentally could be verified. 
TABLE I

INITIAL DESIGN OF THE MACHINE GEOMETRY

\begin{tabular}{lcc}
\hline \hline Variable & Value & Unit \\
\hline$D_{\mathrm{ro}} / D_{\mathrm{ri}}$ & $460 / 430$ & $\mathrm{~mm}$ \\
$D_{\mathrm{so}} / D_{\mathrm{si}}$ & $419 / 228$ & $\mathrm{~mm}$ \\
$l_{s}$ & 100 & $\mathrm{~mm}$ \\
$A_{\text {slot }}$ & 802.7 & $\mathrm{~mm}$ \\
$L_{m}$ & 8 & $\mathrm{~mm}$ \\
$\delta_{g}$ & 0.6 & $\mathrm{~mm}$ \\
$S_{w}$ & 15 & $\mathrm{~mm}$ \\
$P_{\text {arc }}$ & 100 & ${ }^{\mathrm{e}}$ \\
$S_{d}$ & 50 & $\mathrm{~mm}$ \\
$Q_{s}$ & 36 & \\
$\alpha_{p}$ & 0.55 & \\
$2 P$ & 40 & \\
$m$ & 3 & \\
\hline \hline
\end{tabular}

X. K. Gao et al. [5] investigatedM optimization using RSM with a zoom-in windows method and mixed-resolution CCD (MR-CCD) experiment design. A complex and modern design function, mixed-resolution CCD has been theoretically presented. The article lacks comparative results to show the advantages of the proposed robust optimization method.

In X. Liu and H. W. Fu's paper [6], a dynamic dual-RSM was studied to optimize copper losses, torque, and efficiency. All mentioned references were studies on PM synchronous machines. This article used also a complex model, where particle swarm and RSM (using radial basis function) methods are employed to improve torque and efficiency. Although, the work suffers an experimental investigation.

In this study, a novel approach is applied, where a modified Booth's multiplication algorithm utilizing simulated annealing (BA-SA) is used to develop design optimization process of electrical machines. Simulated annealing (SA) is a methodology for solving unconstrained and bound-constrained optimization issues. SA results from statistical thermal physics and simulates the cooling process of a material from its melting-state at a high temperature to its crystallization-state at a low temperature. This physical process can be used to develop a new nonlinear optimization method, the SA algorithm, in which the model parameters are observed as state vectors and the objective function is observed as an energy function of the physical system. The nature of SA can be referred to as a heuristic Monte Carlo method with a higher efficiency and effectiveness [7], [8].

In this paper, a multi-objective optimization is employed to improve the output power performance and the SPMSG's' cost. The electromagnetic behavior of a SPMSG is investigated by various key design parameters through DRSM with different design optimization approaches and the analysis of variance (ANOVA) method, where the MR-RD as a modern design function is proposed. Following this, sorted results are used in the BA-SA to determine the optimum point, but not necessarily the perfect point to meet both objectives of maximizing output power and minimizing materials cost.

\section{Initial Design AND PRoblem Definition}

The objective of this study relies on a permanent magnet synchronous AC generator with exterior rotor topology applied to a wind turbine. The initial design parameters are given in Table I. The stator contains 36 segmented teeth with closed slot

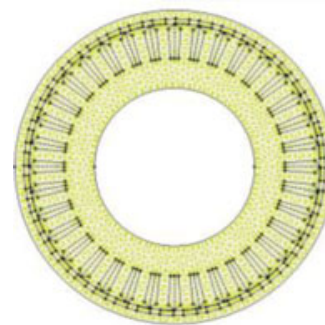

(a)

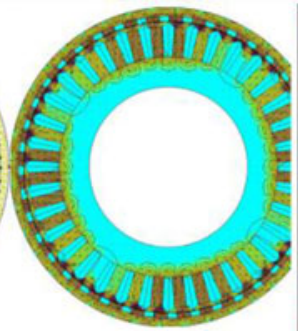

(b)

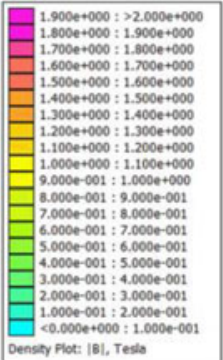

Fig. 1. Schematic of the SPMSG, a) generated mesh, and b) magnetic-field distribution using FEA.

TABLE II

DESIGN CONTROLLABLE TREATMENTS

\begin{tabular}{ccccc}
\hline \hline & Controllable design treatments & \multicolumn{3}{c}{ Coded design treatments } \\
\hline & All variables are normalized & -1 & 0 & 1 \\
$x_{1}$ & Magnet thickness (mm), $l_{m}$ & 5 & 6.5 & 8 \\
$x_{2}$ & Air-gap length (mm), $l_{g}$ & 0.5 & 1.17 & 2 \\
$x_{3}$ & Slot width (mm), $S_{w}$ & 8 & 12 & 15.5 \\
\hline \hline
\end{tabular}

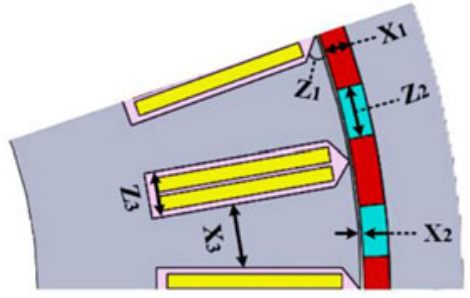

Fig. 2. Design parameters of the studied SPMSG.

modulation in order to reduce the amplitude of cogging torque significantly (Fig. 1). A 2D FE model is studied to examine of magnetic flux distribution. The mesh is automatically generated [Fig. 1(a)], and the magnetic field distribution is shown in Fig. 1(b).

The objective of the design is to maximize the output power while remaining cost-effective. Table I demonstrates the design parameters of a 1st-level RSM optimization, in which the current density fixed as $1.22 \mathrm{~A} / \mathrm{mm}^{2}$. Based on Table II and Fig. 2 define, the three main design treatments (controllable variables). Also, the three noise variables for the MR-RD approach are considered, in which the under-cut angle of the stator tooth tip $\left(\delta_{\mathrm{st}}\right)$ changes between $25^{\circ} \leq \mathrm{z}_{1} \leq 45^{\circ}$, air-gap between PMs $\left(L_{\mathrm{a}-\mathrm{pm}}\right)$ which varies between $3 \mathrm{~mm} \leq \mathrm{z}_{2} \leq 5 \mathrm{~mm}$, and the tooth width between $10 \mathrm{~mm} \leq \mathrm{z}_{3} \leq 20 \mathrm{~mm}$.

The BBD, CCD, MR-TD and FFD approaches are calculated with $13,15,25,27$ runs, respectively.

Fig. 3 shows how the pre-analysis and post-analysis are linked to provide the optimum operation point for output power maximization with an acceptable performance. The FEA uses LUA scripting to analyze the electromagnetic performance of the SPMSG. Afterwards, finalized output data from the design block is integrated with the multi-objective optimization block (yellow block) to find the best set the treatment combinations through DRSM using the least squares method (LSM) with different design functions. Then, Booth's algorithm using SA is used to minimize the cost of the materials and maximize the output power. 


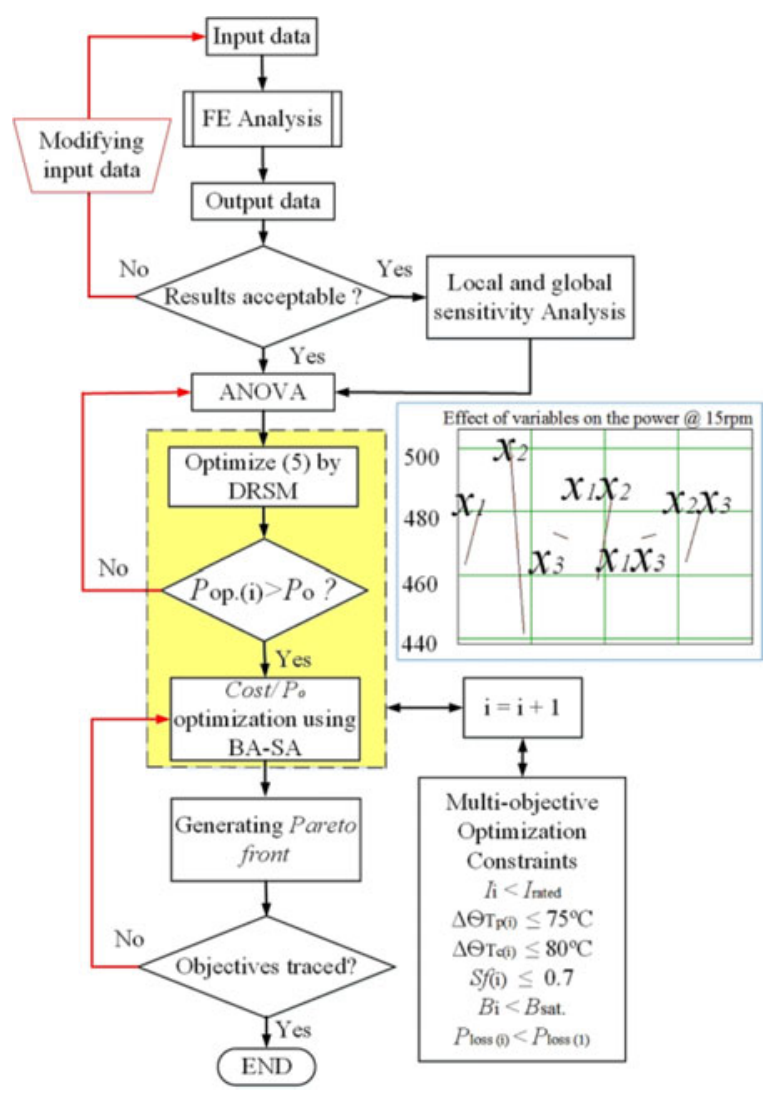

Fig. 3. Flowchart of the proposed multi-objective design optimization.

\section{DuAl-LEVEl Response Surface Methodology}

DRSM is a multi-level collection of mathematical and statistical techniques used to detect the "best-fit" using the MR-RD, $\mathrm{BBD}, \mathrm{CCD}$, and FFD approaches. The analysis is performed in order to observe the effect of a number of chosen variables on a quantity of interest. The objective is always to optimize the response in the physical system. Since the model integrates a curvature in the system to approximate the response, this study requires the second-order polynomial model as given

$$
\begin{gathered}
y_{1}=\beta_{0}+\sum_{i=1}^{k} \beta_{i} x_{i}+\sum_{i=1}^{k} \beta_{i i} x_{i}^{2}+\sum_{i<j} \sum^{\beta_{i j}} x_{i} x_{j}+\varepsilon_{i j} \\
x=\left[\begin{array}{c}
x_{1} \\
x_{2} \\
\vdots \\
x_{k}
\end{array}\right], b=\left[\begin{array}{c}
\widehat{\beta}_{1} \\
\widehat{\beta}_{2} \\
\vdots \\
\widehat{\beta}_{k}
\end{array}\right], \& B=\left[\begin{array}{cccc}
\widehat{\beta}_{11} & \widehat{\beta}_{12} / 2 & \ldots & \widehat{\beta}_{1 k} / 2 \\
\ldots & \widehat{\beta}_{22} & \ldots & \widehat{\beta}_{2 k} / 2 \\
\ldots & \ldots & \ldots & \ldots \\
s y m & \ldots & \ldots & \widehat{\beta}_{k k}
\end{array}\right]
\end{gathered}
$$

where $x$ and $b$ represent a $(k \times 1)$ vector of the treatments and first-order regression coefficients, respectively. $B$ is a $(k \times k)$ symmetrical matrix that consists of pure quadratic coefficients $\left(\widehat{\beta}_{i i}\right)$ in the main diagonal elements, and for off- diagonal elements, one-half the mixed quadratic coefficients $\widehat{\beta}_{i j}, i \neq j . \varepsilon$ is the error observed in the response of the first-level $y_{1} \cdot \varepsilon_{\mathrm{ij}}$ integrates any other sources of variability in the experiment consisting of measurement variability arising from noise and differences among units [9].

A normalization of treatments is needed in order to eliminate unit differences and prevent rounding errors by next regression

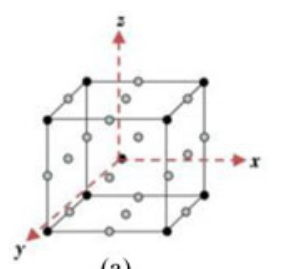

(a)

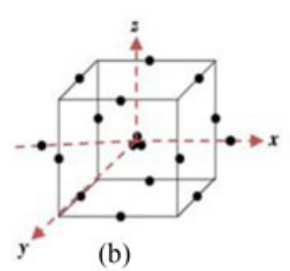

(b)

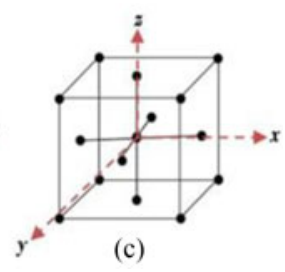

(c)
Fig. 4. The DOE models using, a) FFD, b) CCD, and c) BBD approaches.

analysis. Each treatment $\left(\zeta_{i}\right)$ can be coded with a variable $\left(x_{i}\right)$ via its maximum and minimum as follows

$$
\begin{aligned}
x_{i} & =\frac{\zeta_{i}-\bar{\zeta}_{i}}{\Delta \zeta_{i}} \\
\bar{\zeta}_{i} & =\frac{\zeta_{i \max }+\zeta_{i \min }}{2} \text { and } \Delta \zeta_{i}=\frac{\zeta_{i \max }-\zeta_{i \min }}{2}
\end{aligned}
$$

\section{A. Mixed Resolution Robust Design (MR-RD)}

The MR-RD approach is used to improve the quality of yields and processes by 25 runs, and has as the defining relation $I=x_{1} x_{2} x_{3} z_{1}=z_{1} z_{2} z_{3}=x_{1} x_{2} x_{3} z_{2} z_{3}$. The quality of results are improved, whilst a higher level of performance is obtained via three controllable and three uncontrollable variables. The highest possible performance is obtained by determining the optimum combination of design treatments [10]. The consistency of performance can be improved by making the yield/process insensitive to uncontrollable treatments (known as noise). In this approach, the optimum design should be determined by using design of experiment (DOE) principles. Also, performance consistency is achieved by carrying out the experiment under the influence of the noise treatments. The DOE using the MR$\mathrm{RD}$ approach can economically satisfy the needs of problem solving and yield/process design optimization of electrical machines as long as the number of runs is significantly reduced. This results in a large emphasis on size and cost of experiments. Additionally, the challenge arises in obtaining this response due to the influence of the uncontrollable noise factors. Sets of noise factors, by adding variability to the system, effect the target response [11], [12]. The function model of this design is validated in $[10]$.

\section{B. The Full Factorial Design (FFD)}

This type of design is known as the most expensive experimental design. It consists of the largest number of experiment which consider only controllable treatments $\left(3^{3}\right)=27$ runs. FFD refers to the three levels of treatments as low, intermediate, and high $\left(x_{1}, x_{2}, x_{3}\right)$ as needed to observe the true response $\eta^{\prime}$, which consists of an experimental error (or noise) known as $\varepsilon_{\text {exp }}$. This error is given by the measurement block with a variance $\left(\sigma^{2}\right)$. In geometrical terms, the observations should be simulated on the vertex of a cube, in the middle of its faces and edges, and at the origin. The cube model of this design is illustrated in Fig. 4(a) [4].

\section{The Central Composite Design (CCD)}

A Box-Wilson Central Composite Design, generally referred to as a central composite design (CCD), is studied by many statisticians in the RSM. It is perhaps the most popular class of 
TABLE III

ANOVA TABLE OF THE FitTEd Model

\begin{tabular}{lccc}
\hline \hline $\begin{array}{l}\text { Source of } \\
\text { Variation }\end{array}$ & $\begin{array}{c}\text { Degree of } \\
\text { freedom }\end{array}$ & $\begin{array}{c}\text { Sum of } \\
\text { Squares (SS) }\end{array}$ & $\begin{array}{c}\text { Mean } \\
\text { Square }\end{array}$ \\
\hline Regression & $p-1$ & $S S R=\sum_{i=1}^{n}\left(\widehat{y}_{i}-\bar{y}\right)^{2}$ & $S S R /(p-1)$ \\
Residual (Error) & $N-p$ & $S S E=\sum_{i=1}^{n}\left(y_{i}-\widehat{y}_{i}\right)^{2}$ & $S S E /(N-p)$ \\
Total & $N-1$ & $S S T=\sum_{i=1}^{n}\left(y_{i}-\bar{y}\right)^{2}$ & \\
\hline \hline
\end{tabular}

TABLE IV

AdD / SUBtract Signed-MAgNitude GeNERATOR

\begin{tabular}{lcccc}
\hline \hline \multirow{2}{*}{ Operation } & \multirow{2}{*}{ Add Magnitudes } & \multicolumn{3}{c}{ Subtract Magnitudes } \\
\cline { 3 - 5 } & & $\mathrm{A}>\mathrm{B}$ & $\mathrm{A}<\mathrm{B}$ & $\mathrm{A}=\mathrm{B}$ \\
\hline$(+\mathrm{A})+(+\mathrm{B})$ & $+(\mathrm{A}+\mathrm{B})$ & & & \\
$(+\mathrm{A})+(-\mathrm{B})$ & & $+(\mathrm{A}-\mathrm{B})$ & $-(\mathrm{B}-\mathrm{A})$ & $+(\mathrm{A}-\mathrm{B})$ \\
$(-\mathrm{A})+(+\mathrm{B})$ & & $-(\mathrm{A}-\mathrm{B})$ & $+(\mathrm{B}-\mathrm{A})$ & $+(\mathrm{A}-\mathrm{B})$ \\
$(-\mathrm{A})+(-\mathrm{B})$ & $-(\mathrm{A}+\mathrm{B})$ & & & \\
$(+\mathrm{A})-(+\mathrm{B})$ & $+(\mathrm{A}+\mathrm{B})$ & $+\mathrm{A}-\mathrm{B})$ & $-(\mathrm{B}-\mathrm{A})$ & $+(\mathrm{A}-\mathrm{B})$ \\
$(+\mathrm{A})-(-\mathrm{B})$ & $+(\mathrm{B}+\mathrm{B})$ & & & \\
$(-\mathrm{A})-(+\mathrm{B})$ & $-(\mathrm{A}-\mathrm{B})$ & $+(\mathrm{B}-\mathrm{A})$ & $+(\mathrm{A}-\mathrm{B})$ \\
$(-\mathrm{A})-(-\mathrm{B})$ & & - &
\end{tabular}

second order designs. The design involves 15 runs that consist of the eight vertices of the first cube at $( \pm 1, \pm 1, \pm 1)$ as cube points, and six star points at $( \pm 1.682,0,0),(0, \pm 1.682,0)$, and $(0,0, \pm 1.682)$ plus the origin of the fist cube $(0,0,0)$. After the first-level optimization by the first cube, the origin of the second cube (second-level) is provided by the optimum points of the observation $\left(\mathrm{y}_{1}\right)$. The cube model of this design is illustrated in Fig. 4(b) [4].

\section{The Box-Behnken Design (BBD)}

The BBD is well known as a subset of the FFD, and is the most economical design due to only requiring 13 runs, which is less than other conventional designs such as FFD and CCD. Therefore, it is comparable to the design of MR-RD only if its error is as small as that of MR-RD. In terms of complexity, it can be called the simplest type of design in comparison to the others. This is considered when these experiments cannot be realized for practical reasons such as issues with physical constraints. The cube model of the design is illustrated in Fig. 4(c) [13].

Table III presents the analysis-of-variance (ANOVA) results, which can be used to check the adequacy of fit in the design region for each level of optimization. The observation of this table is output power of the generator [14].

\section{BOOTh's Algorithm Using SimUlated ANNEALING (SA)}

Booth's Algorithm (BA) is a method particularly applicable for multiplying signed numbers integrated by SA to solve electrical machine issues in this study. This begins with the addition and subtraction of the signed-magnitudes in different configurations (Table IV), as there are multiple ways to compute a yield. BA is a well-known multiplication algorithm, which utilizes two complementary notations of signed binary numbers for multiplication. BA is also called a uniform shift method which examines multiple portions of the multiplier simultaneously to

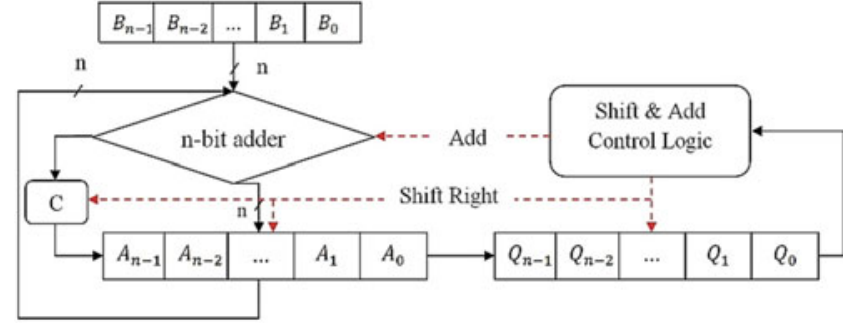

Fig. 5. The general flowchart of modified N-Bit bus Booth's algorithm.

determine multiples of the multiplicand to be added to the partial yield. This algorithm requires no sign correction for the two complementary notation numbers and the decoding of the multiplier may initiate from either direction. The main drawback of the algorithm is that it still relies on a process which needs $n$ shifts and an average of $n / 2$ additions for an n-bit multiplier. A boosted multiplication speed can be reached by evaluating more than two bits of the multiplier at a time as the process can be simply seen with an $n$-bit multiplier (Fig. 5). In this figure, $B_{0}$ to $B_{n-1}$ stand for the multiplicand process (MP), $C$-block is a 1-bit register block, $Q_{0}$ to $Q_{n-1}$ represent the multiplier process and the dashed lines show control signal orders [15], [16].

In this study, a novel method, known as Simulated Annealing (SA), was integrated with Booth's algorithm. SA is a method for solving unconstrained and bound-constrained optimization issues. The method models the physical process of heating a material and afterward slowly lowering the temperature to reduce defects, thus minimizing the system energy. At each iteration of the SA method, a new point is randomly generated. The distance of the new point from the current point, or the extent of the search, is based on a probability distribution with a scale proportional to the temperature. The algorithm accepts all new points which lower the objective, but also, with a certain probability, points which raise the objective. Through accepting points that raise the objective, the algorithm avoids being trapped at local minima, and is able to search globally for more possible solutions. An Annealing schedule is selected to systematically reduce the cost of the SPMSG as the algorithm proceeds. As the temperature decreases, the algorithm reduces the extent of its search to converge to a minimum cost of the SPMSG.

The objective function, validated in [17], is defined as

$$
\text { s.t. }\left\{\begin{array}{l}
f(x)=\frac{C}{C_{0}}+\frac{P_{0}}{P} \\
g_{1}(x)=0.92-\eta \leq 0 \\
g_{2}(x)=450-P_{\text {out }} \leq 0 \\
g_{3}(x)=J_{c}-4 \leq 0 \\
g_{4}(x)=\Delta \theta T_{p}-75 \leq 0 \\
g_{5}(x)=\Delta \theta T_{c}-80 \leq 0 \\
g_{6}(x)=B_{i}-2.1 \leq 0 \\
g_{7}(x)=s f-0.7 \leq 0
\end{array}\right.
$$

where $C_{0}$, and $P_{0}$ stand for current cost of design and output power of the generator, and $C$ and $P$ are the optimum values of $C_{0}$ and $P_{0}$, respectively. Under these conditions, the efficiency is greater than $92 \%$, the output power $>450 \mathrm{~W}$ at a minimum speed $(15 \mathrm{rpm})$, and the current density $\left(J_{c}\right)$ should not exceed $4 \mathrm{~A} / \mathrm{mm}^{2}$ [3]. The probability of acceptance by SA can be given by Equation (6) where $\Delta$ shows [new objective - previous objective], and $T$ stands for the current temperature.

$$
S_{a}=1 / 1+\exp (\Delta / \max (T))
$$




\section{OPTIMIZATION AND ANALYSIS}

In this section, a discussion is presented on how the multiobjective optimization, with an aim of maximization of output power in conjunction with a minimization of the generator's cost, will be investigated. This will be approached through a multi-disciplined study of the generator's cost where the results of optimization will be discussed.

\section{A. Optimization Constraints}

To consider the local constrained optimization of the $d$-axis current at each operating point based on [18], for objective $P_{\text {opt.(i) }}$ in each operating point, the $d$-axis current $i_{d, i}$ should be evaluated. To complete the description of this optimization problem, a number of conditions are required to be defined. Some constraints must be satisfied at each operating point. They can be qualified as instantaneous constraints:

a) Rated current: $\forall i, \sqrt{i_{d, i}^{2}+i_{q, i}^{2}} \leq \sqrt{3} \cdot I_{r}$

b) Rated voltage: $\forall i, \sqrt{v_{d, i}^{2}+v_{q, i}^{2}} \leq \sqrt{3} \cdot V_{\text {rated }}$

c) EM saturation for each part of the machine as

$$
\forall i, B_{i}(\max ) \leq B_{\text {sat. }}
$$

d) The losses evaluation by $\forall i, P_{\text {loss }, i}<P_{\text {loss }}$

e) Magnet and winding temperature-rise by

$$
\begin{aligned}
& \max \left(\Delta \theta T_{P M}\right)_{i} \leq\left(\Delta \theta T_{P M}\right)_{\max } \\
& \max \left(\Delta \theta T_{c}\right)_{i} \leq\left(\Delta \theta T_{c}\right)_{\max }
\end{aligned}
$$

where maximum induction level $B_{i}(\max )$ is $1.9 \mathrm{~T}$. Each operating point of the profile is treated independently, and current control is optimized at every operating point to not only minimize the PMSM's drive losses but also satisfy the conditions above. A current control strategy (direct and transverse axis current) is used to contribute to the extension of the constant speed power range, which is validated in [16]. This consideration takes the form of an $\left(i_{d}, i_{q}\right)$ optimization to minimize losses at each operating point of the profile respecting other instantaneous constraints (a, b, c, and d) [19]. Therefore, $i_{d, i}$ should be optimized, otherwise the particular operating point cannot be reached. The instantaneous constraints $\mathrm{c}$ and $\mathrm{d}$ are satisfied at each operating point $\left(T_{i}, \Omega_{i}\right)$, where $B_{\mathrm{sat}}$ and also demagnetization, are subject to being smaller than $2.1 \mathrm{~T}$ and $891 \times 10^{3} \mathrm{~A} . \mathrm{m}^{-1}$, respectively. The thermal constraint requires additional consideration. The temperature-rise of the hottest point relative to ambient temperature must be calculated accurately by means of a transient thermal model. The maximum value over the entire temperature-rise profile must therefore plateau below a threshold value, $\max \left(\Delta \theta \mathrm{T}_{\mathrm{P}}(\mathrm{t})\right) \leq 75^{\circ} \mathrm{C}$, and $\max \left(\Delta \theta \mathrm{T}_{c}(\mathrm{t})\right) \leq 80^{\circ} \mathrm{C}$ [20]. Although, the thermal transient analysis is very time consuming, the following two definitions are considered based on thermal time constants (TCs) to allow the simulation run via steady-state thermal analysis:

a) While TCs is large: $\sum_{i} \Delta \theta_{i} d_{i} / \sum_{i} d_{i}$

b) While TCs is small: $\operatorname{Max}(\Delta \theta(\mathrm{t}))$

$\Delta \theta$ is calculated once in the winding, and another time in the magnet that corresponds to the temperature-rise between the hottest point (of the slot or magnet) as well as the ambient temperature. The copper loss with respect to the armature resistance per phase $\left(R_{s}\right)$ resolves through the set of geometrical parameters. The copper resistivity is chosen at the maximum temperature $\left(80^{\circ} \mathrm{C}\right)[21],[22]$. Hence, total copper losses per

\begin{tabular}{|c|c|c|c|c|c|c|}
\hline Approach & Source & $\begin{array}{l}\text { Sum of } \\
\text { Squares }\end{array}$ & $\mathrm{DF}$ & $\begin{array}{l}\text { Mean } \\
\text { square }\end{array}$ & $F$ & $R^{2}$ \\
\hline \multirow[t]{5}{*}{ FFD } & A & $1.661 * 10^{3}$ & - & $1.661 * 10^{3}$ & 0.672 & - \\
\hline & B & 162.605 & - & 162.605 & 0.066 & - \\
\hline & $\mathrm{AB}$ & 13.249 & - & 13.249 & $5.36 * 10^{-3}$ & - \\
\hline & Error & $0.8 \%$ & 4 & 2.472 & - & - \\
\hline & Total & $1.173 * 10^{4}$ & 7 & - & - & $99.93 \%$ \\
\hline \multirow[t]{5}{*}{ MR-RD } & A & $1.556 * 10^{3}$ & - & $1.556 * 10^{3}$ & 0.788 & - \\
\hline & B & 143.605 & - & 143.605 & 0.098 & - \\
\hline & $\mathrm{AB}$ & 11.249 & - & 11.249 & $6.79 * 10^{-3}$ & - \\
\hline & Error & $0.77 \%$ & 4 & 3.130 & - & - \\
\hline & Total & $1.453 * 10^{4}$ & 6 & - & - & $99.94 \%$ \\
\hline \multirow[t]{5}{*}{$\mathrm{CCD}$} & A & $1.597 * 10^{3}$ & - & $1.556 * 10^{3}$ & 0.799 & - \\
\hline & B & 168.605 & - & 143.605 & 0.1 & - \\
\hline & $\mathrm{AB}$ & 11.997 & - & 11.249 & $6.98 * 10^{-3}$ & - \\
\hline & Error & $0.91 \%$ & 4 & 3.130 & - & - \\
\hline & Total & $1.503 * 10^{4}$ & 8 & - & - & $99.81 \%$ \\
\hline \multirow[t]{5}{*}{$\mathrm{BBD}$} & A & $1.669 * 10^{3}$ & - & $1.556 * 10^{3}$ & 0.799 & - \\
\hline & B & 173.781 & - & 143.605 & 0.1 & - \\
\hline & $\mathrm{AB}$ & 12.765 & - & 11.249 & $6.98 * 10^{-3}$ & - \\
\hline & Error & $0.88 \%$ & 5 & 3.130 & - & - \\
\hline & Total & $1.803 * 10^{4}$ & 12 & - & - & $99.89 \%$ \\
\hline
\end{tabular}

TABLE V

ANOVA USING DIFFERENT APPROACHES WITH 5\% LEVEL

phase with slot-filling factor of 0.4 can be calculated using

$$
P_{\text {copper }, i}=R_{s}\left(i_{d, i}^{2}+i_{q, i}^{2}\right)
$$

Based on [20], [21] the first harmonic hypothesis, the iron loss can be predicted using (8)

$$
P_{\text {iron }, i}=\left(\frac{2 k_{H}}{\pi}\right)\left|\Omega_{i}\right| \widehat{B}_{i}^{2}+\frac{\left(p \cdot \Omega_{i} \widehat{B}_{i}\right)^{2}}{2} \cdot \alpha_{p}
$$

where $\widehat{B}_{i}$ is the maximum local flux density for $i$ th operating point. $k_{\mathrm{H}}$, and $\alpha_{p}$ are loss coefficients which are 0.045 A.m/V and 73 A.m/V.s respectively. Thus, the total iron loss consists of the iron loss from the machine parts (stator yoke, and teeth). Let us assume that the loss densities are considered to be homogeneous in each of these two parts, while through a static analysis, iron losses at the rotor yoke are nearly zero in the first harmonic.

Table V shows that $R^{2}$ values using FFD, MR-RD, CCD, and BBD are 0.9993, 0.9994, 0.9981, and 0.9989, respectively. As an example, the $R^{2}$ value using FFD represents $99.93 \%$ of the total variation of output power performance. Furthermore, the MR-RD approach results in the minimum error $(0.77 \%)$, whereas the CCD approach results in the maximum value of the residual (error). Therefore, ANOVA is performed to examine how well the fitted-model is able to predict the response at any observation points. For this reason the SST, $F$-value, and $R^{2}$, which resulted from the variation of the response, are calculated. The average errors of the fitted-models of the various design functions are reported as nearly the same across all functions but slightly larger for the model fitted with the CCD function. However, this design function explored a larger domain of the design treatment space than other design models during the observation-stage; it is notable to say that the accuracy of a model is not necessarily increased by increasing the number of experiments involved in the model fit. Fig. 8 indicates the current behavior, and best function values (of (5)) which is analyzed using BA-SA to determine the best function value through 3000 sample iterations in which it was assumed that the efficiency, output power, and current density had to be greater than $92 \%$, greater than $450 \mathrm{~W}$, and less than $4 \mathrm{~A} / \mathrm{mm}^{2}$, respectively. 


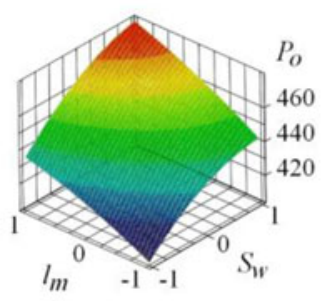

(a)

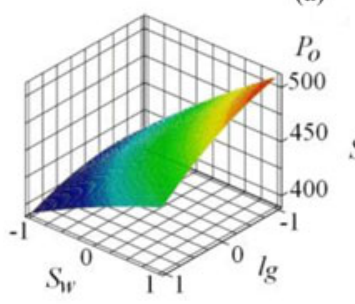

(c)
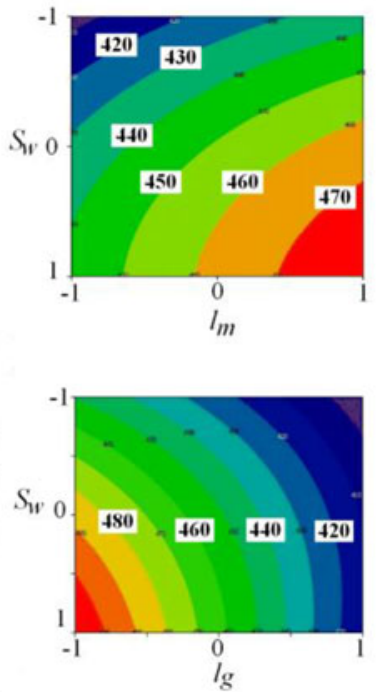

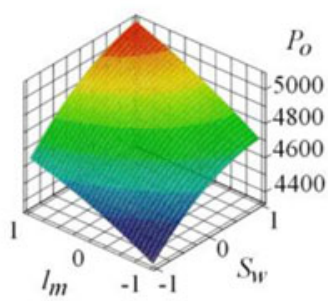

(b)
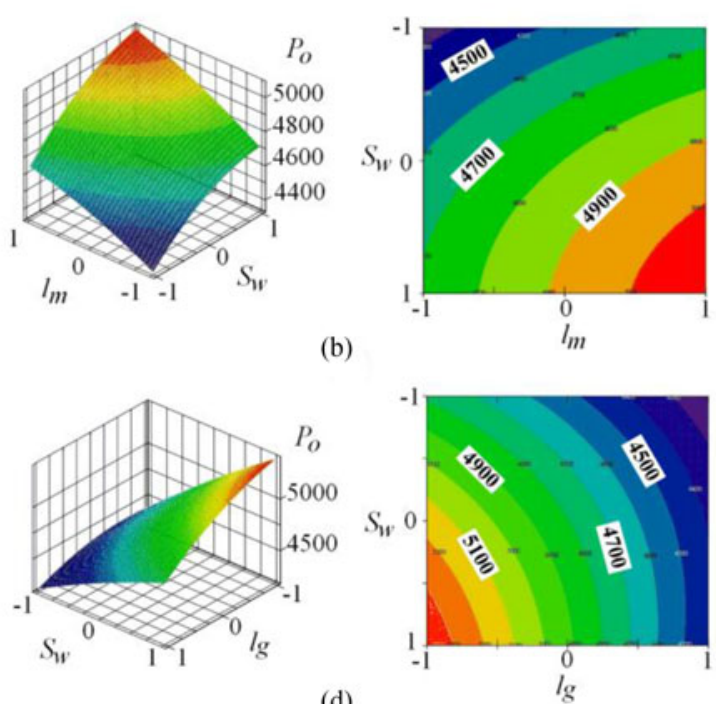

Fig. 6. Surface and counter plots of Multi-level RSM results on $P_{\mathrm{o}}$ objective at minimum speed ( $\left.15 \mathrm{rpm}\right)$ and (150 rpm), a) thickness of magnet versus slot-width @15 rpm, b) thickness of magnet versus slot-width@150 rpm, c) slot-width versus length of magnet @15 rpm, d) slot-width versus length of magnet @150 rpm.

TABLE VI

LSA AND GSA RESULTS

\begin{tabular}{lccccccc}
\hline \hline Par. & \multicolumn{3}{c}{ Amplitude variations of parameter } & \multirow{2}{*}{ Sen. } & \multirow{2}{*}{ Combined sen. } \\
\cline { 2 - 5 } & $-20 \%$ & $-10 \%$ & 0 & $10 \%$ & $20 \%$ & & \\
\hline$l_{m}$ & 3.5 & 4.1 & 4.9 & 5.5 & 6.1 & 0.0995 & 0.1992 \\
$\delta_{g}$ & 1.2 & 1.4 & 1.6 & 1.8 & 2.0 & 0.0991 & 0.2017 \\
$\rho$ & 3.8 & 4.3 & 4.8 & 5.4 & 6.2 & 0.1489 & 0.2081 \\
$S_{w}$ & 2.3 & 2.7 & 3.2 & 3.6 & 4.0 & 0.0744 & 0.2055 \\
$D_{\mathrm{si}}$ & 19.6 & 21.4 & 23.7 & 24.5 & 27.6 & 0.0221 & 0.0791 \\
$D_{\mathrm{ro}}$ & 15.1 & 17.6 & 19.2 & 21.8 & 23.1 & 0.0115 & 0.0685 \\
$l_{\mathrm{P}}$ & 3.1 & 4.5 & 5.5 & 6.8 & 7.6 & 0.0498 & 0.2031 \\
$N_{c}$ & 0.5 & 0.6 & 0.7 & 0.8 & 0.9 & 0.0093 & 0.0284 \\
\hline \hline
\end{tabular}

The influence of the geometrical design parameters on the output power is examined by local/global sensitivity analysis in Table VI. To provide accurate results, each parameter is assumed to have four variations. The table presents the samples required for the data analysis of LSA.

In total, 33 samples are needed in this calculation, in which 32 points are for those four-variations of the initial sample ("0") and the eight parameters. The sensitivity order can be obtained from the data as below

$$
|\rho|>\left|l_{m}\right|>\left|\delta_{g}\right|>\left|S_{w}\right|>\left|l_{p}\right|>\left|D_{s i}\right|>\left|D_{\text {ro }}\right|>\left|N_{c}\right|
$$

To set the optimization framework, the following four factors, $\rho, l_{m}, \delta_{g}$, and $S_{w}$ are considered significant factors.

The second-level RSM plots, presented in Fig. 6, are used to check the variation of the treatments under objective tracking. Also, the counter plots of each surface plot are reported to summarize the results of the study. The RSM plots indicate the response surface based on the defined treatments through two different levels of rotation speed: minimum speed (15 rpm), which is used in the optimization process, and rated speed (150 rpm). The maximum output power that could be generated is $5.1 \mathrm{~kW}$ @ $150 \mathrm{rpm}$, and the minimum generated output power provided is $500 \mathrm{~W}$ @15 rpm.

Based on the fitted model for the RSM, the prediction of the output power can be compared to FEA data in order to check

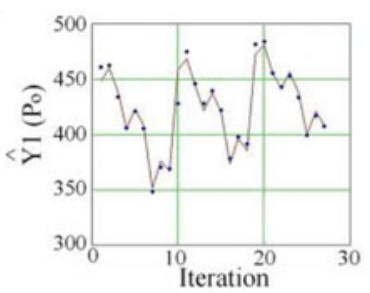

(a)

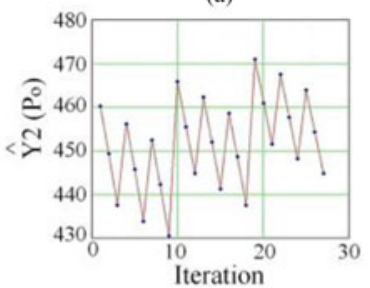

(c)

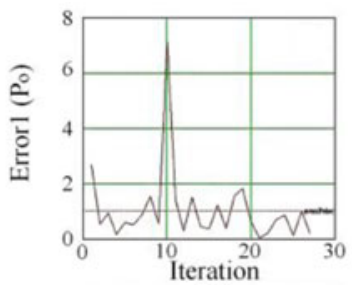

(b)

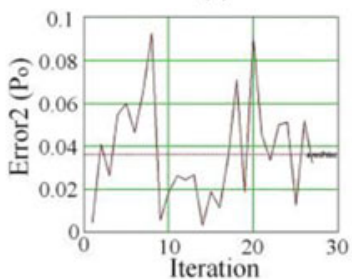

(d)
Fig. 7. Predicted objective $\left(P_{\mathrm{o}}\right)$ via a) 1st-leve fitted-model and FEA data, b) the error of the 1st-leve fitted-model, c) 2nd-leve fitted-model and FEA data, and d) the error of the 1st-leve fitted-model.

the error at $15 \mathrm{rpm}$ as represented in Fig. 7. This demonstrates that the prediction of the fitted model has a small error and can be incorporated into the DRSM. In the first and second-levels of RSM, the calculated second-order regression model can be reported (Table. VIII) based on (1). Fig. 7 demonstrates how the fitted model is improved through the following equation

$$
\begin{aligned}
\widehat{y} & =460.235+5.878 \mathrm{x}_{1}-3.804 \mathrm{x}_{2}-10.8 \mathrm{x}_{3}+0.14 \mathrm{x}_{1} \cdot \mathrm{x}_{2} \\
& +0.76 \mathrm{x}_{1} \cdot \mathrm{x}_{3}+0.09 \mathrm{x}_{2} \cdot \mathrm{x}_{3}-0.286 \mathrm{x}_{1}^{2}+0.017 \mathrm{x}_{2}^{2}-0.247 \mathrm{x}_{3}^{2}
\end{aligned}
$$

The average error is decreased as can be seen in Fig. 7(d) which uses (11).

$$
\text { Error }_{a v e}=\frac{1}{27} \sum_{i=1}^{27}\left|\frac{y_{i}-\widehat{y}_{i}}{y_{i}}\right|
$$




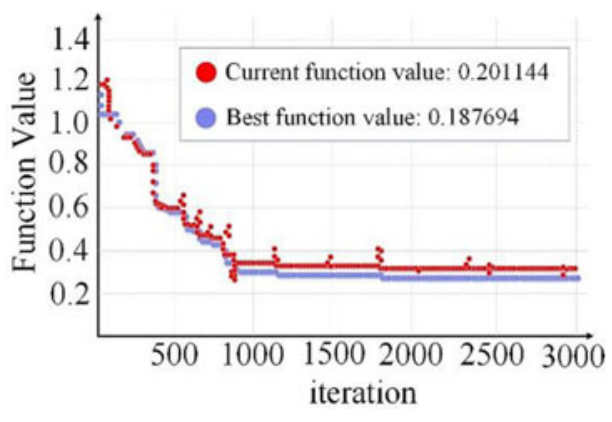

Fig. 8. Cost effective optimization analysis using BA-SA.

TABLE VII

CAlculated Second-Order Regression Model at 1ST-LEVEl AND 2ND-LEVEL RSM @15 RPM

\begin{tabular}{lcc}
\hline \hline Coefficients & First-level RSM & Second-level RSM \\
\hline$\beta_{0}$ & 448.484 & 460.235 \\
$\beta_{1}$ & 7.633 & 5.878 \\
$\beta_{2}$ & -37.811 & -3.804 \\
$\beta_{3}$ & 28.387 & -10.8 \\
$\beta_{4}$ & 6.086 & 0.14 \\
$\beta_{5}$ & -1.714 & 0.76 \\
$\beta_{6}$ & 6.355 & 0.09 \\
$\beta_{7}$ & 2.235 & -0.286 \\
$\beta_{8}$ & -5.305 & 0.017 \\
$\beta_{9}$ & -16.747 & -0.247 \\
\hline \hline
\end{tabular}

In Table VII, the error decreases from 1 to less than 0.04 on average by the 2 nd level coefficients. Thus, a finalized fitted second-order regression model can be given by (10). The fitted regression model is checked via the $F$-value to ascertain the validity under the null hypothesis as

$$
F=\operatorname{SSR}(1-a)^{-1} / \operatorname{SSE}(N-a)^{-1}
$$

where $a$ stands for the number of terms at the fitted-model. Additionally, $R^{2}$ is a significant statistic to express the proportion of the variation of the $y_{i}$ by the fitted-model and FEA data for the mean of $\bar{y}$, as given

$$
R^{2}=S S R \times(S S T)^{-1}
$$

The major design outputs such as $L_{d}, L_{q}, \lambda_{m}, P_{\mathrm{opt}}, \eta \%$, CPSR, $T_{r}$, and $C$ are calculated over two steps of analysis as pre-optimization and post-optimization analyzes using different design functions in which the improvements of multi-objective optimization using DRSM and BA-SA resulted in a minimum rotation speed $(15 \mathrm{rpm})$ in Table VIII. Afterward, FEA method is verified with an insignificant error. In addition, the CPSR is highly sensitive to the machine key parameters. Furthermore, to further improve the accuracy of the optimization, the choice of materials can be studied while taking cost-effective design into consideration.

The procedure can be repeated on a smaller range of design treatments centered on the optimal design that was obtained in this paper.

Fig. 9, demonstrates the multi-objective optimization for determining the optimum design point using Pareto front, in which the best optimum design point is marked at $15 \mathrm{rpm}$ resulting in the maximum output power, and minimum cost of manufacturing that is all summarized in Table VIII. Fig. 10 presents the
TABLE VIII

OPTIMIZATION RESULTS AND VERIFICATION

\begin{tabular}{lcccccc}
\hline \hline Par. & Pre-Opti. & Opti-MR-RD & Opti-FFD & Opti-CCD & Opti-BBD & Test \\
\hline$L_{d}(\mathrm{mH})$ & 468.2 & 470.7 & 471.6 & 468.5 & 469.8 & 473.2 \\
$L_{q}(\mathrm{mH})$ & 468.2 & 471 & 470.9 & 469.7 & 469.2 & 474.5 \\
$D_{\mathrm{si}}(\mathrm{mm})$ & 228 & 234 & 235.6 & 232 & 233 & 234 \\
$D_{\mathrm{si}}(\mathrm{mm})$ & 460 & 452 & 451.6 & 449.8 & 450.4 & 452 \\
$\lambda_{m}(\mathrm{mVs})$ & 1691.8 & 1720.4 & 1714 & 1710 & 1713 & 1799.2 \\
$P_{\mathrm{o}}(\mathrm{W})$ & 440.7 & 514.8 & 507.2 & 488 & 498 & 511.6 \\
$\eta(\%)$ & 92.2 & 96.6 & 94.8 & 93.7 & 94.3 & 97 \\
$C P S R$ & 434.7 & 497.8 & 477.3 & 469 & 470 & 505.7 \\
$l_{g}$ & 1.0 & 0.9 & 0.88 & 0.8 & 0.95 & 0.9 \\
$N_{c}(\mathrm{turn})$ & 120 & 80 & 98 & 111.8 & 110 & 80 \\
$\rho\left(\mathrm{g} / \mathrm{m}{ }^{3}\right)$ & 7.63 & 8.5 & 8.3 & 7.96 & 8.1 & 8.5 \\
$l_{\mathrm{p}}(\mathrm{mm})$ & 100 & 92 & 95 & 94.3 & 90 & 92 \\
$T_{r}$ & 314.6 & 370.35 & 367.4 & 348 & 356.5 & 370 \\
Cost $(\$)$ & 2979.6 & 2000.6 & 2480 & 2880 & 2690.5 & 2000.6 \\
\hline \hline
\end{tabular}

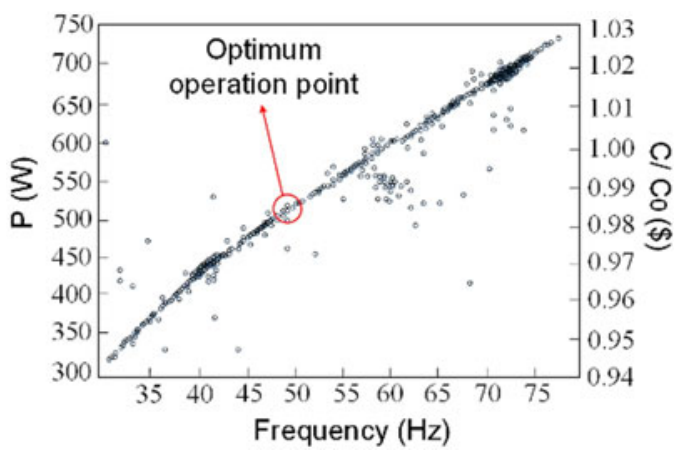

Fig. 9. Pareto front of the multi-objective optimization at various frequency.

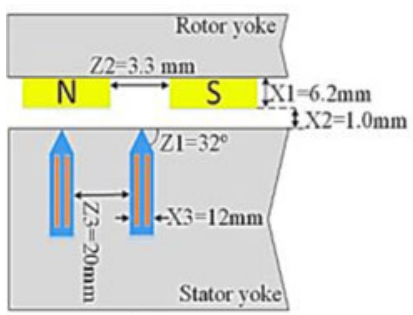

(a)

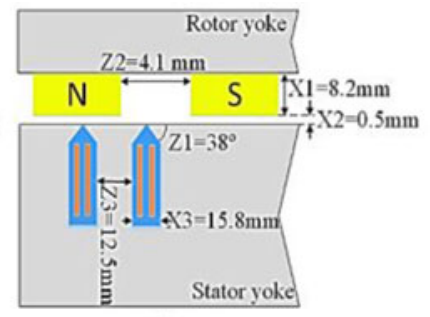

(b)
Fig. 10. Controllable and uncontrollable variables on a) the initial and b) optimized model.

values of the controllable and uncontrollable design variables on the initial model (Fig. 10(a)), and the impact of these variables on the machine's structure after multi-objective optimization shown in Fig. 10(b).

\section{EXPERIMENTAL INVESTIGATION}

The manufactured prototype is tested for the case of small power generation (5 kW) via wind energy along the coast of Barcelona in Spain. Since the primary objective relies on the maximization of the output power, the measured output power in comparison to the initial, optimized, and FE models can be seen in Fig. 11. In this figure, a notable accuracy between the optimized generator and measured values presents the best fit of the optimization design. The optimized SPMSG in the test was fed via a variable speed frequency converter (ABB ACS600) and loaded by a DC machine. The electrical output 


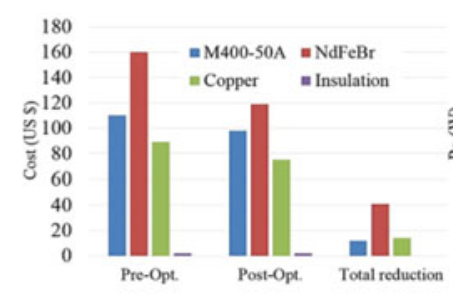

(a)

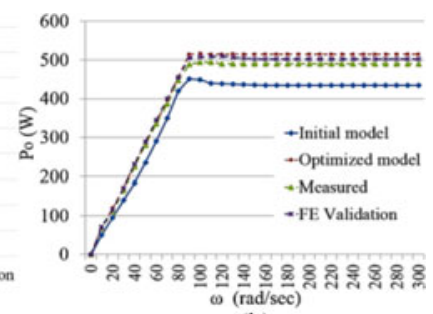

(b)
Fig. 11. A comparison over output power and cost objectives, a) Cost, and b) output power with simulated and experimental data.
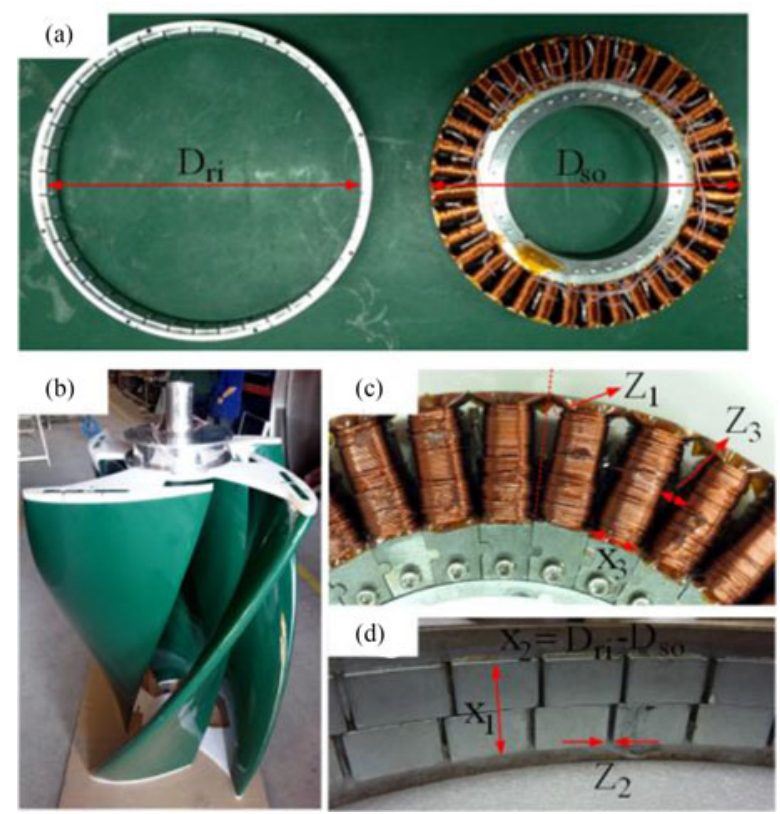

Fig. 12. The manufactured analyzed model with variables definition, a) the SPMSG with segmented stator and rotor cores, b) installed VAWT on the generator, c) variables on the stator, and d) variables on the rotor cores.

power is measured by a Yokogawa PZ4000 with an accuracy of $1 \%$, and the temperature is registered in twenty points by Pt-100 temperature sensors. The manufactured SPMSG can be seen in Fig. 12(a) with a segmented stator core for improving slot fill factor, simpler winding, and closed-slot topology, the rotor included the magnets. This prototype is used for wind energy production using a vertical-axis twisted savonius type wind turbine (VAWT) is installed on the SPMSG, shown in Fig. 12(b), and the controllable and uncontrollable variables demonstration on the stator and rotor cores in Fig. 12(c)-(d).

\section{CONCLUSION}

In this research, two different optimization methods are employed to deal with a multi-objective design function, a mathematical and statistical technique (DRSM using zoom-in- window approach), and a multiplication-algorithm-based (BA-SA). The objective is to maximize the output power and minimize manufacturing cost under a number of optimization constraints. This comparative study applied various types of design functions to find the best-fit parametric model, in which the influence of chosen design parameters is evaluated by local and global sensitivity analysis along with ANOVA. MR-RD with a minimum error $(0.77 \%)$ is proposed, which is used for the very first time in the machine's design. The objectives of the research were achieved, where pre-optimization and post-optimization results were compared and FEA validation, with an error less than $0.5 \%$, corresponded with analytical and mathematical calculations. Measured experimental results were additionally reported that support the improvement of the design optimization.

\section{REFERENCES}

[1] H. Fang and D. Wang, "A novel design method of permanent magnet synchronous generator from perspective of permanent magnet material saving," IEEE Trans. Energy Conv., vol. 32, no. 1, pp. 48-53, Mar. 2017.

[2] D. C. Montgomery, Design and Analysis of Experiments. Singapore: Wiley, 2013, pp. 65-544.

[3] G. Lei et al., Multidisciplinary Design Optimization Methods for Electrical Machines and Drive Systems. New York, NY, USA: Springer-Verlag, 2016, pp. 73-103.

[4] L. Jolly et al., "Design optimization of permanent magnet motors using response surface methodology and genetic algorithms," IEEE Trans. Magn., vol. 41, no. 10, pp. 3928-3930, Oct. 2005.

[5] X. K. Gao et al., "Robust design for torque optimization using response surface methodology," IEEE Trans. Magn., vol. 38, no. 2, pp. 1-4, Mar. 2002.

[6] X. Liu and W. N. Fu, "A dynamic dual-response-surface methodology for optimal design of a permanent-magnet motor using finite-element method," IEEE Trans. Magn, vol. 52, no. 3, pp. 1-4, Mar. 2016.

[7] L. P. Rubinfield, "A proof of the modified booth's algorithm for multiplication,” IEEE Trans. Comput., vol. C-24, no. 10, pp. 14-15, Oct. 1975.

[8] D. Bertsimas and J. Tsitsiklis, "Simulated annealing," J. Stat. Sci., vol. 8, no. 1, pp. 10-15, 1993.

[9] L. Jolly et al., "Design optimization of permanent magnet motors using response surface methodology and genetic algorithms," IEEE Trans. Magn., vol. 41, no. 10, pp. 3928-3930, Oct. 2005.

[10] T. J. Robinson et al., "Robust parameter design: A review," Reliab Eng. Int., vol. 39, no. 1, pp. 90-98, 2004, doi: 10.1002/qre.602.

[11] J. J. Borkowski and J. M. Lucas, "Designs of mixed resolution for process robustness studies," TECHNOMETRICS, vol. 39, no. 1, pp. 1-8, 1997.

[12] C. M. Borror and D. C. Montgomery, "Mixed resolution designs as alternatives to Taguchi inner/outer array designs for robust design problems," Qual. Reliab. Eng. Int., vol. 16, no. 2, pp. 117-127, 2000.

[13] S.-H. Lee et al., "Multiobjective optimization design of small-scale wind power generator with outer rotor based on box-behnken design," IEEE Trans. App. Supercond., vol. 26, no. 4, pp. 1-5, Jun. 2016.

[14] W. Li and C. Su, "Modeling and optimization of acid chrome dark blue degradation by fenton using box-Behnken response surface methodology," in Proc. Int. Conf. Mech. Autom. Control Eng., 2011, pp. 7463-7465.

[15] J.-H. Hong and C.-W. Wu, "Cellular-array modular multiplier for fast RSA public-key cryptosystem based on modified Booth's algorithm," IEEE Trans. VLSI Syst., vol. 11, no. 3, pp. 474-481, Jun. 2003.

[16] E. Ambikairajah and M. J. Carery, "Technique for performing multiplication on a 16-bit microprocessor using an extension of booth's algorithm,' IEEE Electron. Lett., vol. 16, no. 2, pp. 53-54, Jan. 1980.

[17] G. Lei, C. Liu, J. Zhu, and Y. Guo, "Techniques for multilevel design optimization of permanent magnet motors," IEEE Trans. Energy Conv., vol. 30, no. 4, pp. 1574-1582, Dec. 2015.

[18] L. Chedot et al., "Integrated starter generator: The need for an optimal design and control approach. Application to a permanent magnet machine,' IEEE Trans. Ind. Appl., vol. 43, no. 2, pp. 552-559, Mar./Apr. 2007.

[19] S. Morimoto et al., "Expansion of operating limits for permanent magnet motor by current vector control considering inverter capacity," IEEE Trans. Ind. Appl., vol. 26, no. 5, pp. 866-871, Sep. 1990.

[20] J. Aubry et al., "Sizing optimization methodology of a surface permanent magnet machine-converter system over a torque-speed operating profile: Application to a wave energy converter," IEEE Trans. Ind. Electron. vol. 59, no. 5, pp. 2116-2124, May 2012.

[21] C. Mademlis, J. Xypteras, and N. Margaris, "Loss minimization in surface permanent-magnet synchronous motor drives,' IEEE Trans. Ind. Electron., vol. 47, no. 1, pp. 115-122, Feb. 2000.

[22] C. Cavallaro, A. O. DiTommaso, R. Miceli, A. Raciti, G. Galluzzo, and M. Trapanese, "Efficiency enhancement of permanent-magnet synchronous motor drives by online loss minimization approaches," IEEE Trans. Ind. Electron., vol. 52, no. 4, pp. 1154-1160, Aug. 2005. 